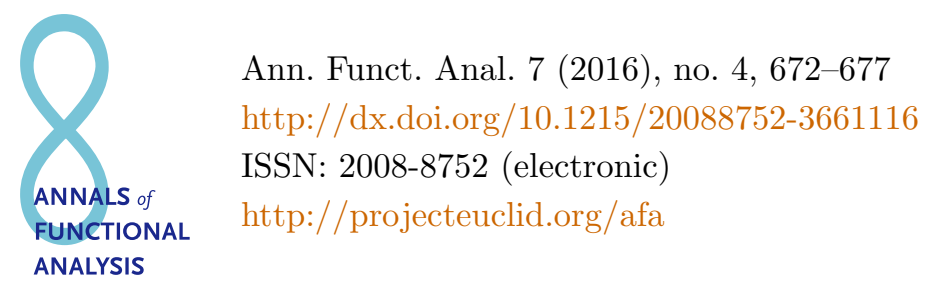

\title{
A NEW CHARACTERIZATION OF THE BOUNDED APPROXIMATION PROPERTY
}

\author{
JU MYUNG KIM ${ }^{1}$ and KEUN YOUNG LEE ${ }^{2 *}$
}

Communicated by J. Esterle

\begin{abstract}
We prove that a Banach space $X$ has the bounded approximation property if and only if, for every separable Banach space $Z$ and every injective operator $T$ from $Z$ to $X$, there exists a net $\left(S_{\alpha}\right)$ of finite-rank operators from $Z$ to $X$ with $\left\|S_{\alpha}\right\| \leq \lambda_{T}$ such that $\lim _{\alpha}\left\|S_{\alpha} z-T z\right\|=0$ for every $z \in Z$.
\end{abstract}

\section{INTRODUCTION}

A Banach space $X$ is said to have the approximation property (AP) if, for every compact subset $K$ of $X$ and every $\varepsilon>0$, there exists a finite-rank and continuous linear map (operator) $S$ from $X$ to $X$ such that $\sup _{x \in K}\|S x-x\| \leq \varepsilon$; briefly, $\operatorname{id}_{X} \in \overline{\mathcal{F}(X, X)}{ }^{\tau_{c}}$, where $\operatorname{id}_{X}$ is the identity map on $X, \mathcal{F}(X, X)$ is the space of all finite-rank operators from $X$ to $X$, and $\tau_{c}$ is the topology of uniformly compact convergence on the ideal $\mathcal{L}$ of all operators. If there exists a $\lambda \geq 1$ such that $\operatorname{id}_{X} \in$ $\{S \in \mathcal{F}(X, X):\|S\| \leq \lambda\}{ }^{\tau_{c}}$, then we say that $X$ has the bounded approximation property (BAP) or $\lambda$-BAP when we need to indicate the constant $\lambda$.

Lima, Nygaard, and Oja [5, Corollary 1.5] proved that $X$ has the AP if and only if, for every Banach space $Y$ and every $T \in \mathcal{W}(Y, X)$, the space of all weakly compact operators from $Y$ to $X, T \in \overline{\{S \in \mathcal{F}(Y, X):\|S\| \leq\|T\|\}^{\tau_{c}}}$. A simple verification shows that $X$ has the BAP if and only if for every Banach space $Y$ and every $T \in \mathcal{L}(Y, X)$, there exists a $\lambda_{T}>0$ such that $T \in\left\{S \in \mathcal{F}(Y, X):\|S\| \leq \lambda_{T}\right\}^{\tau_{c}}$.

Copyright 2016 by the Tusi Mathematical Research Group.

Received Mar. 22, 2016; Accepted Jun. 24, 2016.

${ }^{*}$ Corresponding author.

2010 Mathematics Subject Classification. Primary 46B28; Secondary 47L20.

Keywords. bounded approximation property, bounded compact approximation property, separable Banach space. 


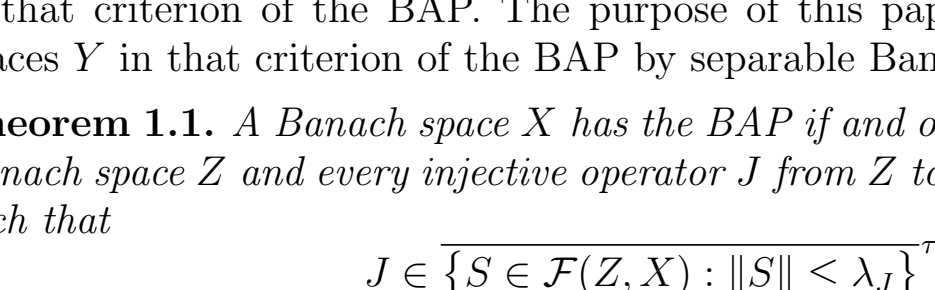

\title{
A NEW CHARACTERIZATION OF THE BOUNDED APPROXIMATION PROPERTY
}

\author{
JU MYUNG KIM ${ }^{1}$ and KEUN YOUNG LEE ${ }^{2 *}$
}

Communicated by J. Esterle

\begin{abstract}
We prove that a Banach space $X$ has the bounded approximation property if and only if, for every separable Banach space $Z$ and every injective operator $T$ from $Z$ to $X$, there exists a net $\left(S_{\alpha}\right)$ of finite-rank operators from $Z$ to $X$ with $\left\|S_{\alpha}\right\| \leq \lambda_{T}$ such that $\lim _{\alpha}\left\|S_{\alpha} z-T z\right\|=0$ for every $z \in Z$.
\end{abstract}

\section{INTRODUCTION}

A Banach space $X$ is said to have the approximation property (AP) if, for every compact subset $K$ of $X$ and every $\varepsilon>0$, there exists a finite-rank and continuous linear map (operator) $S$ from $X$ to $X$ such that $\sup _{x \in K}\|S x-x\| \leq \varepsilon$; briefly, $\operatorname{id}_{X} \in \overline{\mathcal{F}(X, X)}{ }^{\tau_{c}}$, where $\operatorname{id}_{X}$ is the identity map on $X, \mathcal{F}(X, X)$ is the space of all finite-rank operators from $X$ to $X$, and $\tau_{c}$ is the topology of uniformly compact convergence on the ideal $\mathcal{L}$ of all operators. If there exists a $\lambda \geq 1$ such that $\operatorname{id}_{X} \in$ $\{S \in \mathcal{F}(X, X):\|S\| \leq \lambda\}{ }^{\tau_{c}}$, then we say that $X$ has the bounded approximation property (BAP) or $\lambda$-BAP when we need to indicate the constant $\lambda$.

Lima, Nygaard, and Oja [5, Corollary 1.5] proved that $X$ has the AP if and only if, for every Banach space $Y$ and every $T \in \mathcal{W}(Y, X)$, the space of all weakly compact operators from $Y$ to $X, T \in \overline{\{S \in \mathcal{F}(Y, X):\|S\| \leq\|T\|\}^{\tau_{c}}}$. A simple verification shows that $X$ has the BAP if and only if for every Banach space $Y$ and every $T \in \mathcal{L}(Y, X)$, there exists a $\lambda_{T}>0$ such that $T \in\left\{S \in \mathcal{F}(Y, X):\|S\| \leq \lambda_{T}\right\}^{\tau_{c}}$.

Copyright 2016 by the Tusi Mathematical Research Group.

Received Mar. 22, 2016; Accepted Jun. 24, 2016.

${ }^{*}$ Corresponding author.

2010 Mathematics Subject Classification. Primary 46B28; Secondary 47L20.

Keywords. bounded approximation property, bounded compact approximation property, separable Banach space. 


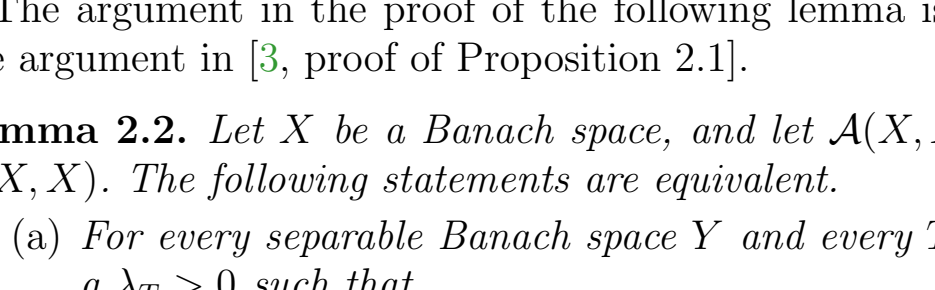

\title{
A NEW CHARACTERIZATION OF THE BOUNDED APPROXIMATION PROPERTY
}

\author{
JU MYUNG KIM ${ }^{1}$ and KEUN YOUNG LEE ${ }^{2 *}$
}

Communicated by J. Esterle

\begin{abstract}
We prove that a Banach space $X$ has the bounded approximation property if and only if, for every separable Banach space $Z$ and every injective operator $T$ from $Z$ to $X$, there exists a net $\left(S_{\alpha}\right)$ of finite-rank operators from $Z$ to $X$ with $\left\|S_{\alpha}\right\| \leq \lambda_{T}$ such that $\lim _{\alpha}\left\|S_{\alpha} z-T z\right\|=0$ for every $z \in Z$.
\end{abstract}

\section{INTRODUCTION}

A Banach space $X$ is said to have the approximation property (AP) if, for every compact subset $K$ of $X$ and every $\varepsilon>0$, there exists a finite-rank and continuous linear map (operator) $S$ from $X$ to $X$ such that $\sup _{x \in K}\|S x-x\| \leq \varepsilon$; briefly, $\operatorname{id}_{X} \in \overline{\mathcal{F}(X, X)}{ }^{\tau_{c}}$, where $\operatorname{id}_{X}$ is the identity map on $X, \mathcal{F}(X, X)$ is the space of all finite-rank operators from $X$ to $X$, and $\tau_{c}$ is the topology of uniformly compact convergence on the ideal $\mathcal{L}$ of all operators. If there exists a $\lambda \geq 1$ such that $\operatorname{id}_{X} \in$ $\{S \in \mathcal{F}(X, X):\|S\| \leq \lambda\}{ }^{\tau_{c}}$, then we say that $X$ has the bounded approximation property (BAP) or $\lambda$-BAP when we need to indicate the constant $\lambda$.

Lima, Nygaard, and Oja [5, Corollary 1.5] proved that $X$ has the AP if and only if, for every Banach space $Y$ and every $T \in \mathcal{W}(Y, X)$, the space of all weakly compact operators from $Y$ to $X, T \in \overline{\{S \in \mathcal{F}(Y, X):\|S\| \leq\|T\|\}^{\tau_{c}}}$. A simple verification shows that $X$ has the BAP if and only if for every Banach space $Y$ and every $T \in \mathcal{L}(Y, X)$, there exists a $\lambda_{T}>0$ such that $T \in\left\{S \in \mathcal{F}(Y, X):\|S\| \leq \lambda_{T}\right\}^{\tau_{c}}$.

Copyright 2016 by the Tusi Mathematical Research Group.

Received Mar. 22, 2016; Accepted Jun. 24, 2016.

${ }^{*}$ Corresponding author.

2010 Mathematics Subject Classification. Primary 46B28; Secondary 47L20.

Keywords. bounded approximation property, bounded compact approximation property, separable Banach space. 


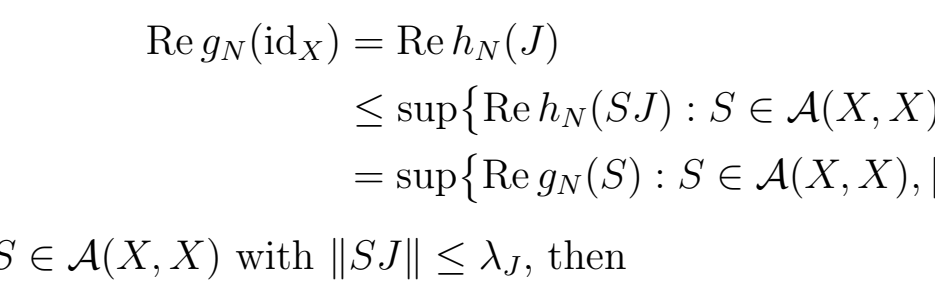

\title{
A NEW CHARACTERIZATION OF THE BOUNDED APPROXIMATION PROPERTY
}

\author{
JU MYUNG KIM ${ }^{1}$ and KEUN YOUNG LEE ${ }^{2 *}$
}

Communicated by J. Esterle

\begin{abstract}
We prove that a Banach space $X$ has the bounded approximation property if and only if, for every separable Banach space $Z$ and every injective operator $T$ from $Z$ to $X$, there exists a net $\left(S_{\alpha}\right)$ of finite-rank operators from $Z$ to $X$ with $\left\|S_{\alpha}\right\| \leq \lambda_{T}$ such that $\lim _{\alpha}\left\|S_{\alpha} z-T z\right\|=0$ for every $z \in Z$.
\end{abstract}

\section{INTRODUCTION}

A Banach space $X$ is said to have the approximation property (AP) if, for every compact subset $K$ of $X$ and every $\varepsilon>0$, there exists a finite-rank and continuous linear map (operator) $S$ from $X$ to $X$ such that $\sup _{x \in K}\|S x-x\| \leq \varepsilon$; briefly, $\operatorname{id}_{X} \in \overline{\mathcal{F}(X, X)}{ }^{\tau_{c}}$, where $\operatorname{id}_{X}$ is the identity map on $X, \mathcal{F}(X, X)$ is the space of all finite-rank operators from $X$ to $X$, and $\tau_{c}$ is the topology of uniformly compact convergence on the ideal $\mathcal{L}$ of all operators. If there exists a $\lambda \geq 1$ such that $\operatorname{id}_{X} \in$ $\{S \in \mathcal{F}(X, X):\|S\| \leq \lambda\}{ }^{\tau_{c}}$, then we say that $X$ has the bounded approximation property (BAP) or $\lambda$-BAP when we need to indicate the constant $\lambda$.

Lima, Nygaard, and Oja [5, Corollary 1.5] proved that $X$ has the AP if and only if, for every Banach space $Y$ and every $T \in \mathcal{W}(Y, X)$, the space of all weakly compact operators from $Y$ to $X, T \in \overline{\{S \in \mathcal{F}(Y, X):\|S\| \leq\|T\|\}^{\tau_{c}}}$. A simple verification shows that $X$ has the BAP if and only if for every Banach space $Y$ and every $T \in \mathcal{L}(Y, X)$, there exists a $\lambda_{T}>0$ such that $T \in\left\{S \in \mathcal{F}(Y, X):\|S\| \leq \lambda_{T}\right\}^{\tau_{c}}$.

Copyright 2016 by the Tusi Mathematical Research Group.

Received Mar. 22, 2016; Accepted Jun. 24, 2016.

${ }^{*}$ Corresponding author.

2010 Mathematics Subject Classification. Primary 46B28; Secondary 47L20.

Keywords. bounded approximation property, bounded compact approximation property, separable Banach space. 


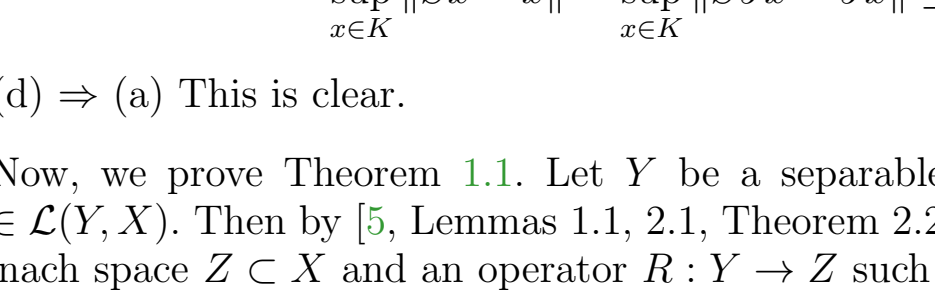

\title{
A NEW CHARACTERIZATION OF THE BOUNDED APPROXIMATION PROPERTY
}

\author{
JU MYUNG KIM ${ }^{1}$ and KEUN YOUNG LEE ${ }^{2 *}$
}

Communicated by J. Esterle

\begin{abstract}
We prove that a Banach space $X$ has the bounded approximation property if and only if, for every separable Banach space $Z$ and every injective operator $T$ from $Z$ to $X$, there exists a net $\left(S_{\alpha}\right)$ of finite-rank operators from $Z$ to $X$ with $\left\|S_{\alpha}\right\| \leq \lambda_{T}$ such that $\lim _{\alpha}\left\|S_{\alpha} z-T z\right\|=0$ for every $z \in Z$.
\end{abstract}

\section{INTRODUCTION}

A Banach space $X$ is said to have the approximation property (AP) if, for every compact subset $K$ of $X$ and every $\varepsilon>0$, there exists a finite-rank and continuous linear map (operator) $S$ from $X$ to $X$ such that $\sup _{x \in K}\|S x-x\| \leq \varepsilon$; briefly, $\operatorname{id}_{X} \in \overline{\mathcal{F}(X, X)}{ }^{\tau_{c}}$, where $\operatorname{id}_{X}$ is the identity map on $X, \mathcal{F}(X, X)$ is the space of all finite-rank operators from $X$ to $X$, and $\tau_{c}$ is the topology of uniformly compact convergence on the ideal $\mathcal{L}$ of all operators. If there exists a $\lambda \geq 1$ such that $\operatorname{id}_{X} \in$ $\{S \in \mathcal{F}(X, X):\|S\| \leq \lambda\}{ }^{\tau_{c}}$, then we say that $X$ has the bounded approximation property (BAP) or $\lambda$-BAP when we need to indicate the constant $\lambda$.

Lima, Nygaard, and Oja [5, Corollary 1.5] proved that $X$ has the AP if and only if, for every Banach space $Y$ and every $T \in \mathcal{W}(Y, X)$, the space of all weakly compact operators from $Y$ to $X, T \in \overline{\{S \in \mathcal{F}(Y, X):\|S\| \leq\|T\|\}^{\tau_{c}}}$. A simple verification shows that $X$ has the BAP if and only if for every Banach space $Y$ and every $T \in \mathcal{L}(Y, X)$, there exists a $\lambda_{T}>0$ such that $T \in\left\{S \in \mathcal{F}(Y, X):\|S\| \leq \lambda_{T}\right\}^{\tau_{c}}$.

Copyright 2016 by the Tusi Mathematical Research Group.

Received Mar. 22, 2016; Accepted Jun. 24, 2016.

${ }^{*}$ Corresponding author.

2010 Mathematics Subject Classification. Primary 46B28; Secondary 47L20.

Keywords. bounded approximation property, bounded compact approximation property, separable Banach space. 


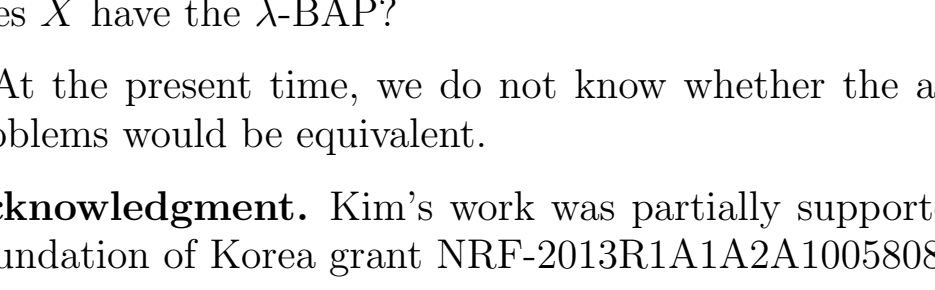

\title{
A NEW CHARACTERIZATION OF THE BOUNDED APPROXIMATION PROPERTY
}

\author{
JU MYUNG KIM ${ }^{1}$ and KEUN YOUNG LEE ${ }^{2 *}$
}

Communicated by J. Esterle

\begin{abstract}
We prove that a Banach space $X$ has the bounded approximation property if and only if, for every separable Banach space $Z$ and every injective operator $T$ from $Z$ to $X$, there exists a net $\left(S_{\alpha}\right)$ of finite-rank operators from $Z$ to $X$ with $\left\|S_{\alpha}\right\| \leq \lambda_{T}$ such that $\lim _{\alpha}\left\|S_{\alpha} z-T z\right\|=0$ for every $z \in Z$.
\end{abstract}

\section{INTRODUCTION}

A Banach space $X$ is said to have the approximation property (AP) if, for every compact subset $K$ of $X$ and every $\varepsilon>0$, there exists a finite-rank and continuous linear map (operator) $S$ from $X$ to $X$ such that $\sup _{x \in K}\|S x-x\| \leq \varepsilon$; briefly, $\operatorname{id}_{X} \in \overline{\mathcal{F}(X, X)}{ }^{\tau_{c}}$, where $\operatorname{id}_{X}$ is the identity map on $X, \mathcal{F}(X, X)$ is the space of all finite-rank operators from $X$ to $X$, and $\tau_{c}$ is the topology of uniformly compact convergence on the ideal $\mathcal{L}$ of all operators. If there exists a $\lambda \geq 1$ such that $\operatorname{id}_{X} \in$ $\{S \in \mathcal{F}(X, X):\|S\| \leq \lambda\}{ }^{\tau_{c}}$, then we say that $X$ has the bounded approximation property (BAP) or $\lambda$-BAP when we need to indicate the constant $\lambda$.

Lima, Nygaard, and Oja [5, Corollary 1.5] proved that $X$ has the AP if and only if, for every Banach space $Y$ and every $T \in \mathcal{W}(Y, X)$, the space of all weakly compact operators from $Y$ to $X, T \in \overline{\{S \in \mathcal{F}(Y, X):\|S\| \leq\|T\|\}^{\tau_{c}}}$. A simple verification shows that $X$ has the BAP if and only if for every Banach space $Y$ and every $T \in \mathcal{L}(Y, X)$, there exists a $\lambda_{T}>0$ such that $T \in\left\{S \in \mathcal{F}(Y, X):\|S\| \leq \lambda_{T}\right\}^{\tau_{c}}$.

Copyright 2016 by the Tusi Mathematical Research Group.

Received Mar. 22, 2016; Accepted Jun. 24, 2016.

${ }^{*}$ Corresponding author.

2010 Mathematics Subject Classification. Primary 46B28; Secondary 47L20.

Keywords. bounded approximation property, bounded compact approximation property, separable Banach space. 